\title{
La raison du plus fort est toujours la meilleure : la représentation du Saint-Siège au Canada, 1877-1917
}

\section{Roberto Perin}

Volume 50, numéro 1, 1983

Bilan de l'histoire religieuse au Canada

Canadian Catholic History: A survey

URI : https://id.erudit.org/iderudit/1007038ar

DOI : https://doi.org/10.7202/1007038ar

Aller au sommaire du numéro

Éditeur(s)

Les Éditions Historia Ecclesiæ Catholicæ Canadensis Inc.

ISSN

0318-6172 (imprimé)

1927-7067 (numérique)

Découvrir la revue

Citer cet article

Perin, R. (1983). La raison du plus fort est toujours la meilleure : la

représentation du Saint-Siège au Canada, 1877-1917. Sessions d'étude - Société canadienne d'histoire de l'Église catholique, 50(1), 99-117.

https://doi.org/10.7202/1007038ar

Tous droits réservés @ Les Éditions Historia Ecclesiæ Catholicæ Canadensis Inc., 1983
Ce document est protégé par la loi sur le droit d'auteur. L'utilisation des services d'Érudit (y compris la reproduction) est assujettie à sa politique d'utilisation que vous pouvez consulter en ligne. 


\section{La raison du plus fort est toujours la meilleure:}

\section{la représentation du Saint-Siège au Canada,}

\section{7-1917}

L'établissement de la délégation apostolique au Canada et ses premières années d'histoire sont liés à l'évolution politique du Québec à la fin du XIX ${ }^{e}$ siècle. En effet, sans la montée du parti libéral au Canada français et sans les accusations d'influence politique qu'il lança contre le clergé, il n'y aurait pas eu de délégation apostolique. Ce fut ce parti qui poussa le Saint-Siège à envoyer $\mathbf{M}^{\mathrm{gr}}$ George Conroy en 1877, $\mathbf{M}^{\mathrm{gr}}$ Rafael Merry del Val vingt ans plus tard et qui, enfin, le détermina à établir une délégation apostolique permanente avec $\mathbf{M}^{\mathrm{gr}}$ Diodeme Falconio comme premier titulaire. Et même après sa défaite, le parti de Laurier continua à influencer la manière de voir du délégué apostolique et, par delà, du Saint-Siège.

Notons qu'en 1877, l'archevêché de Québec fut un puissant appui au parti libéral lorsque celui-ci réclama l'envoi d'un représentant du Saint-Siège au Canada. Vingt ans plus tard, cependant, la situation s'était sensiblement modifiée. Laurier n'eut pas alors l'archevêché de Québec comme allié à Rome. D'ailleurs, il n'en avait pas besoin puisqu'à force de répéter les mêmes plaintes contre le clergé pendant vingt ans, il amena le Saint-Siège à agir par-dessus la tête de l'épiscopat québécois, d'abord en nommant Merry del Val comme délégué apostolique et ensuite en créant une délégation permanente. Au nom de la presque totalité des évêques du Québec, $\mathbf{M}^{\mathrm{gr}}$ Louis-Nazaire Bégin combattit Laurier farouchement, soit au Canada soit à Rome, à cause de la prise de position des libéraux dans la question des écoles du Manitoba et à cause de leurs accusations contre le clergé. L'ironie du sort voulut que le successeur de $\mathbf{M}^{\mathrm{gr}}$ Elzéar Taschereau ait eu recours aux mêmes arguments qu'employèrent à l'époque les évêques Ignace Bourget et Louis Laflèche pour réfuter ces accusations. Bégin n'eut pas cependant l'oreille de Rome et le clergé québécois en vint à se sentir, dans les deux premières décennies de ce siècle, de plus en plus distant de la dé- 
légation apostolique et même du Saint-Siège. On eut nettement l'impression que, Rome, sinon Dieu lui-même, n'était pas du côté des Canadiens français dans ce moment critique de leur histoire.

Sans doute le Saint-Siège prit-il au sérieux ces accusations d'ingérence cléricale en politique à cause de l'agitation menée par l'archevêché de Québec dans les années 1870, agitation qui confirma les plaintes des libéraux et qui servit en même temps à protéger les intérêts de l'archevêché. Ces accusations d'ailleurs ne firent jamais l'objet d'enquête ni de la part de $\mathbf{M}^{\text {gr }}$ Conroy, ni d'aucun autre prélat. Quoi qu'il en soit, Rome se montra implacable sur une seule question pendant ces quarante ans: elle condamna l'intervention apparemment injustifiée du clergé québécois en politique. Or, en se rangeant du côté de $\mathbf{M}^{\mathrm{gr}}$ Taschereau, le Saint-Siège favorisa un homme et son entourage au détriment d'une continuité institutionnelle qui se manifesta par la suite, lorsque $\mathrm{M}^{\mathrm{gr}}$ Bégin reprit presque mot pour mot les arguments des ultramontains les plus exaltés.

Les effets néfastes de cette politique romaine ne se manifestèrent pas à court terme. Mais lorsqu'éclatèrent, à l'époque de Laurier et après, les grandes controverses touchant l'enseignement catholique et le statut du français au Canada, l'Église québécoise se montra incapable de faire valoir ses propres intérêts. Rome s'en remit alors aux hommes politiques, désireux avant tout de se maintenir au pouvoir, et aux ecclésiastiques d'Ontario, plus éloignés des problèmes de l'heure et moins portés à l'action, contrairement au clergé québécois qui avait une conscience historique très aiguë et un instinct politique, dans son sens le plus large, très raffiné. Quoi qu'il en soit, le Saint-Siège, toujours sensible aux accusations d'ingérence cléricale, garda le clergé québécois en bride grâce à l'action du délégué apostolique, et favorisa, malgré lui, l'extinction des droits religieux et linguistiques dans l'Ouest canadien.

\section{LA MISSION CONROY}

Nous avons étudié ailleurs la mission canadienne de $\mathrm{M}^{\mathrm{gr}}$ George Conroy '. Rappelons ici les grandes lignes de cette affaire. Joseph Cauchon, député libéral et président du Conseil privé, en fut l'instigateur. Il se plaignit à deux reprises, en 1876 et en 1877, de l'action politique du clergé québécois auprès du Saint-Siège. En cela il fut secondé par

\footnotetext{
${ }^{1}$ Roberto Perin, «Troppo Ardenti Sacerdoti : the Conroy Mission Revisited», Canadian Historical Review LXI (Sept. 1980) pp. 283-304.
} 
ses amis cléricaux qui étaient aussi conseillers de $\mathbf{M}^{\mathrm{gr}}$ Taschereau. La question «explosive" de l'intervention cléricale en politique au Québec n'était pas nouvelle pour le Saint-Siège. Elle avait déjà fait l'objet d'une intervention du Saint-Office en 1874. Mais, en 1877, la Propagande se crut obligée d'adopter des mesures plus énergiques.

Dans l'esprit de Rome, l'envoi d'un délégué apostolique au Canada devait être une mesure temporaire. Mais ce légat aurait à préparer la voie à des visites ultérieures de représentants romains. Il était donc crucial qu'il établisse de bons rapports avec les autorités civiles. La nationalité du candidat n'était pas indifférente pour les cardinaux de la Propagande: le délégué devait être italien ou au moins britannique ${ }^{2}$. Son mandat serait d'essayer de résoudre une foule de controverses qui déchiraient l'Église québécoise à cette époque. Cependant le Saint-Siège insista surtout sur la question politique qui fut la seule où la ligne de conduite du délégué fut tracée d'avance. On lui demanda d'imposer un silence rigoureux au clergé sur la question politique et de mettre un terme à la censure cléricale du parti libéral, "persécution" que Rome prit pour avérée ${ }^{3}$. Au Canada, $\mathrm{M}^{\mathrm{gr}}$ Conroy suivit fidèlement ses instructions. Il noua des rapports cordiaux avec le gouverneur général Dufferin, un Irlandais comme Conroy, ainsi qu'avec le gouvernement d'Alexander Mackenzie. Cependant, il serait injuste de prétendre, comme le firent à l'époque les plus zélés des disciples de Bourget, qu'il était dupe des déclarations d'orthodoxie religieuse des libéraux. Il ne se gêna pas, par exemple, pour condamner devant Mackenzie l'école libérale démocratique de son parti, qui se réclamait de Papineau et qui avait produit selon lui de «très mauvais» discours ${ }^{4}$.

Il reste que Conroy avait sensiblement la même optique que les autorités civiles sur les rapports entre Église et État. D'après lui, l'Église catholique jouissait de privilèges et d'une liberté exceptionnels au Canada. Aux ecclésiastiques qui réclamaient pour le Québec un statut particulier en tant que seule province catholique, $\mathbf{M}^{\text {gr }}$ Conroy répéta les paroles de Dufferin et de Mackenzie: on ne permettrait pas au Québec de constituer un imperium in imperio ${ }^{5}$. Le clergé devait donc éviter d'entrer en conflit avec un des grands partis politiques, ce qui gâte-

\footnotetext{
${ }^{2}$ Archives de la Propaganda Fide, Rome (APFR) Acta (luglio 1879).

${ }^{3}$ APFR, Lettere e Decreti della Santa Congregazione e Biglieti di Monsignor Segretario (LDB), 1877, ff 170-171.

${ }^{4}$ APFR, Scritture riferite in Congressi (sc), 1877, Conroy à Franchi, 30 novembre 1877.

${ }^{5}$ APFR, Sc 1877, Conroy à Franchi, 30 novembre 1877.
} 
rait fatalement ces rapports privilégiés; d'autant plus que le parti libéral dans son ensemble ne s'était pas montré hostile au catholicisme ${ }^{6}$. Le délégué apostolique attribua la passion du clergé québécois pour la politique partisane à son manque de culture théologique. En conséquence, Conroy prescrivit de rehausser le niveau d'étude des jeunes clercs, de choisir des évêques mieux instruits et de renforcer l'autorité épiscopale face à un bas clergé par trop remuant.

La Propagande fut enchantée de l'oeuvre de Conroy, estimant qu'il avait rendu d'éminents services au Saint-Siège et que son rapport était d'une importance capitale ${ }^{7}$. Les cardinaux se réunirent en juillet 1879 afin de décider si Rome devait établir une délégation permanente au Canada et, le cas échéant, de nommer le nouveau titulaire, $\mathrm{M}^{\mathrm{gr}}$ Conroy étant mort prématurément à la fin de sa mission. Le lieutenant-gouverneur du Québec, Letellier de Saint-Just, le conseil des ministres libéral de la province, ainsi que Rodolphe Laflamme et certains de ses collègues firent pression pour que Rome agisse en ce sens ${ }^{8}$. Ils furent appuyés en ceci par des prêtres du Séminaire de Québec ${ }^{9}$. Les consulteurs de la Propagande recommandèrent à l'unanimité la création d'une délégation apostolique permanente au Canada qui mènerait à terme les politiques de la mission Conroy. Les cardinaux durent cependant surseoir à l'exécution de cette proposition, faute de candidat jugé convenable ${ }^{10}$.

\section{LA MISSION SMEULDERS}

Les litiges au sein de l'Église québécoise ne s'apaisèrent pas pour autant. L'exacerbation devint telle que Louis-Nazaire Bégin, alors prêtre au Séminaire de Québec, écrivit des «ultra-montés»: "Ce n'est pas assez d'aplatir ces gens-là; ils ont toute l'élasticité du caoutchouc; c'est un éreintement en bonne forme qu'il leur faut». Et il ajouta au sujet de $\mathrm{M}^{\mathrm{gr}}$ Laflèche: «Ce serait le temps de l'écrapouter ... il veut agir à sa tête ... est-ce que l'autorité ne pourrait pas agir, elle aussi, à sa tête et l'envoyer in partibus infidelium»" ${ }^{11}$.

\footnotetext{
${ }^{6}$ APFR, Sc 1877B, Dufferin to Conroy, 27 March, 1877. Conroy prit soin de traduire cette lettre en italien et l'expédia à la Propagande, en indiquant son entière approbation.

${ }^{7}$ APFR, Acta (luglio 1879).

${ }^{8}$ APFR, Scritture Riferite in Congregazione Generali (SOCG) luglio 1879, Letellier de Saint-Just à Simeoni, R. Laflamme. C. A. P. Pelletier, F. Langelier, F. X. Marchand, H. Starnes, P. Bachand; A. Chauveau à Simeoni, 21 juillet 1878.

${ }^{9}$ APFR, Sc 1878-8, B. Pâquet à inconnu, 6 mars 1879; SOCG luglio 1879, Hamel à Simeoni, 14 mars 1879.

${ }^{10}$ APFR, Acta (luglio 1879).

${ }^{11}$ APFR, Sc 1882-86, Bégin à Célestin Marquis, 25 janv. 1883.
} 
Face à cette aggravation de la situation ecclésiastique au Québec, le Saint-Siège envoya un commissaire apostolique, Henri Smeulders, cistercien belge vivant à Rome et consulteur à la Propagande. Les pouvoirs accordés à Smeulders étaient inférieurs à ceux de Conroy; ce qui expliquerait son titre subalterne. Sa mission était de dénouer les intrigues qui troublaient la paix de l'Église québécoise, mais sans pour autant s'immiscer dans les questions politiques.

Smeulders arriva au Québec en octobre 1883 et y resta quatorze mois. En général, on loua son impartialité. Seuls les adeptes les plus acharnés de $\mathbf{M}^{\mathrm{gr}}$ Laflèche, tel Luc Désilets, le tenaient en suspicion. Cette image dura le temps du séjour de Smeulders dans la capitale qu'il quitta après deux mois pour s'installer définitivement à Montréal. Là il devint un intime de $\mathbf{M}^{\mathrm{gr}}$ Alexandre Taché, qui s'absenta de son diocèse de Saint-Boniface pendant trois mois pour le conseiller.

Tout comme $\mathbf{M}^{\mathrm{gr}}$ Conroy et auparavant $\mathbf{M}^{\mathrm{gr}}$ Ignazio Persico, Smeulders était d'avis que la "médiocrité" de l'épiscopat québécois aggravait une situation déjà difficile. Il déplora la faiblesse de $\mathbf{M}^{\mathrm{gr}}$ Taschereau, reprenant ainsi l'observation de $\mathbf{M}^{\mathrm{gr}}$ Persico, et l'inconstance de $\mathbf{M}^{\mathrm{gr}}$ LouisZéphirin Moreau de St-Hyacinthe de même que celle des frères Antoine et Dominique Racine, respectivement évêques de Sherbrooke et de Chicoutimi. Selon lui, ces trois derniers n'auraient jamais dû accéder à l'épiscopat ${ }^{12}$. Le commissaire réprouva en outre la rigidité de $\mathbf{M}^{\mathrm{gr}}$ Taschereau et de $\mathrm{M}^{\mathrm{gr}}$ Edouard Fabre dans la question universitaire. Leur comportement, opina-t-il, offensait le bon sens et minait l'autorité du Saint-Siège ${ }^{13}$. Cette critique du "rigorisme» épiscopal n'était qu'un écho des sentiments de Conroy qui les avait exprimés à l'endroit d'autres évêques québécois. Enfin, Smeulders et son prédécesseur étaient d'accord pour reconnaître la piété, le dévouement au Saint-Siège et l'attachement à la doctrine religieuse de $\mathbf{M}^{\mathrm{gr}}$ Laflèche.

Sur le fond de la crise ecclésiastique au Québec, les deux représentants de Rome ne partageaient pas les mêmes vues. Smeulders souligna la suffisance de l'archevêque de Québec et de sa 'camarilla' qui voyaient en leurs adversaires des «incultes" ignorants des progrès de la civilisation. "Quoi qu'il en soit, ajouta-t-il, il me semble qu'ils ne sont pas tous des ânes, surtout si l'on considère la qualité des personnes à qui l'on a affaire, parmi lesquelles je retrouve des hommes considérés

${ }^{12}$ APFR, Acta 1884, Smeulders ad Simeoni, 8 aprilis 1884.

${ }^{13}$ APFR, Acta 1884, Smeulders ad Simeoni, 11 februarius 1884. 
comme des sommités du droit, de la magistrature, des avocats renommés qui ont fait leurs cours à Rome. Ce serait plutôt le cas de dire: 'Vox populi, vox Dei'» ${ }^{14}$.

La cause du mal au Canada, d'après Smeulders, était l'existence d'un libéralisme politico-religieux qui refusait de reconnaître que l'État québécois ( «Status Quebecensis») pourrait «en tout faire conformer ses lois aux lois et prescriptions de l'Église» ${ }^{15}$. Cette doctrine néfaste s'était insinuée au Séminaire de Québec où elle avait plusieurs adeptes. Le commissaire proposa en conséquence une épuration du Séminaire, de l'Université Laval et de l'archevêché. Quant à Taschereau, Smeulders suggéra étonnamment qu'on lui confere le chapeau cardinalice et qu'on le retienne à Rome: un séjour prolongé dans la Ville Éternelle aurait pour lui un effet tonifiant ${ }^{16}$.

Bien que prodiguant de bons mots à son commissaire et entérinant certaines de ses recommandations, le Saint-Siège ne partagea pas son interprétation générale de la situation au Canada. En effet, la mission de Smeulders n'infléchit pas la politique globale du Saint-Siège à l'égard du Canada qui resta ce qu'elle était dans les années 1870. Rome distribua ses largesses à l'archevêché de Québec et à ses amis et contint les ennemis de celui-ci. Dans ce contexte il est difficile de voir le démembrement du diocèse de Trois-Rivières autrement que comme une sévère mise en demeure à l'endroit de $\mathbf{M}^{\text {gr }}$ Laflèche et de ses alliés. Quoi qu'il en soit, le climat d'extrême tension au sein de l'Église québécoise se résorba jusqu'au moment où éclata une crise qui fut bien plus grave. Cette longue crise qui commença avec la question des écoles du Manitoba, avait trait au statut de l'enseignement confessionnel et de la langue française au Canada.

\section{LA QUESTION MANITOBAINE ET LA MISSION MERRY DEL VAL}

Parmi les catholiques du Canada, l'archevêque de Saint-Boniface, en collaboration avec l'épiscopat du Québec, joua un rôle de premier plan dans la défense des droits menacés. Au début, Rome encouragea

\footnotetext{
${ }^{14}$ APFR, Acta 1884 , Smeulders à Jacobini, 16 aprile 1884. La plus grande partie de la correspondance des délégués apostoliques est écrite en latin ou surtout en italien. Nos citations sont donc le plus souvent des traductions. Par contre, pour que le lecteur sache à quoi s'en tenir, nous mettons la référence dans la langue d'origine.

${ }^{15}$ APFR, Acta 1884, Smeulders ad Simeoni, 26 aprilis 1884.

${ }^{16}$ APFR, Acta 1884, Smeulders ad Simeoni, 26 junius 1884.
} 
la prudence, mais aussi la fermeté dans ces revendications. Par exemple, le cardinal Mieczyslaw Ledóchowski, préfet de la Propagande, tout en exprimant des réserves à l'égard de la lettre des évêques québécois sur les écoles du Manitoba, les appuya dans leurs pressions politiques. Aux élections de 1896, les libéraux s'en trouvèrent si embarrassés qu'ils décidèrent de mettre un terme à l'engagement politique de l'épiscopat du Québec. Sachant qu'ils n'avaient rien à espérer de la Propagande, les hommes politiques court-circuitèrent le processus décisionnel en s'adressant, à l'aide de puissants alliés anglais, au Secrétariat d'État du Saint-Siège. Tout comme vingt ans auparavant, leur stratégie fut d'accuser le clergé québécois d'immixtion en politique. Ils ressuscitèrent aussi les vieux spectres de guerre religieuse qu'avaient éveillés les prêtres au Séminaire de Québec en 1876. Il n'en fallut pas davantage pour inciter le Saint-Siège à intervenir.

Pour mieux situer l'action de Rome, examinons brièvement les positions respectives des ecclésiastiques québécois et des hommes politiques dans la question scolaire du Manitoba. D'une part, l'épiscopat québécois exigeait une loi fédérale qui, sans rétablir l'état de choses antérieur à l'abolition du système confessionnel, garantirait les droits religieux et linguistiques de la minorité lésée. D'autre part, le leadership libéral, voulant éviter la voie législative qui aurait des conséquences désastreuses pour l'unité du parti et qui présenterait des difficultés constitutionnelles, optait pour une solution négociée qui rendrait aux catholiques manitobains la substance de leurs revendications. Est-il besoin de dire, avec la perspective que nous fournit l'histoire ultérieure, qu'entre la négociation et la législation, la minorité manitobaine aurait été mieux servie par la seconde voie...

Il n'en fut pas ainsi, toutefois. À Rome, les cardinaux de la Congrégation des Affaires ecclésiastiques extraordinaires furent convoqués pour trancher «le problème de l'ingérence du clergé dans la question scolaire au Canada et par conséquence dans les élections politiques»" ${ }^{17}$. On leur assura que les évêques québécois exagéraient les droits constitutionnels de la minorité. Leurs Éminences décidèrent par conséquent d'envoyer un délégué apostolique afin de sortir l'épiscopat québécois du cul-de-sac politique dans lequel leur intransigeance les avait menés. Le délégué devait aussi favoriser un climat de bonne entente entre le clergé et les dirigeants politiques ${ }^{18}$.

\footnotetext{
${ }^{17}$ APFR, Nuova Serie (NS) 240 ( 1902) Rubrica 154, F. Cavagnis, secrétaire de la Congrégation des Affaires ecclésiastiques extraordinaires, à $\mathrm{M}^{\mathrm{gr}}$ Ciasca, secrétaire de la Propagande, 13 gennaio 1897.
}

${ }^{18}$ APFR, NS 240 (1902) Rubrica 154, Cavagnis a Ciasca, 19 febbraio 1897. 
Leur choix tomba sur $\mathrm{M}^{\mathrm{gr}}$ Rafael Merry del Val qui, malgré ses trente-cinq ans, s'était mérité une solide réputation au Secrétariat d'État où il était fonctionnaire. Fils d'aristocrates espagnols, mais formé en Angleterre dans des écoles catholiques huppées, il connaissait parfaitement le fonctionnement du régime parlementaire, condition primordiale, d'après les libéraux, pour une bonne mission au Canada. Merry del Val était aussi ami des Anglais qui avaient plaidé la cause de Laurier en cour de Rome.

Le délégué apostolique suivit ses instructions fidèlement, tâchant d'obtenir par la négociation des réformes à l'accord Laurier-Greenway que le Saint-Siège jugeait insuffisant ${ }^{19}$. Ce faisant, il se buta à l'opposition de tout l'épiscopat québécois, sauf les évêques Emard de Valleyfield et Lorrain, Vicaire apostolique du Pontiac. Il n'est donc pas surprenant que $\mathrm{M}^{\mathrm{gr}}$ Merry del Val ait eu une opinion assez peu flatteuse des prélats du Québec. De $\mathbf{M}^{\mathrm{gr}}$ Langevin de Saint-Boniface, le délégué écrivit: «La démarche empressée de cet excellent prélat nous fait mettre en doute sa prudence" ${ }^{20}$. Cette impression première ne se modifia pas par la suite, bien au contraire.

"La triste situation religieuse existant aujourd'hui au Canada, et surtout dans les provinces de langue française..." ${ }^{21}$ était liée, d'après Merry del Val qui répétait Conroy, au manque de formation du clergé. Comme au temps de la mission Conroy, les jeunes séminaristes servaient de main-d'oeuvre à bon marché dans les collèges classiques de la province et négligeaient ainsi leurs études. Évêques et prêtres s'occupaient de politique et, au lieu d'exercer un leadership social par la supériorité de leur influence morale, ils devenaient les instruments aveugles d'hommes politiques aux intérêts mesquins. Le clergé était tellement impliqué dans la chose publique que, pour faire prévaloir son point de vue, il employait des moyens absolument contraires à la théologie. Le fait que dans plusieurs diocèses le droit canon était lettre morte aggravait cette situation. La seule loi qui souvent y prévalait était la volonté inébranlable de l'évêque. Rappelons ici que cette observation allait tout à fait à l'encontre de ce qu'avait soutenu Conroy vingt ans auparavant à l'effet que le bas clergé affichait une trop grande liberté. Selon Merry

\footnotetext{
${ }^{19}$ Congrégation des Affaires ecclésiastiques extraordinaires (CAEE), Rubricella 37227, «Istruzioni per $\mathrm{M}^{\mathrm{gr}}$ Delegato Apostolico al Canada *, 9 marzo 1897.

${ }^{20}$ CAEE, Inghilterra, Posizione 161 Rubricella 37088, Merry del Val a Rampolla, 30 marzo 1897.

${ }^{21}$ CAEE, Canada, settembre 1897, Appendice, Relazione di $\mathbf{M}^{\text {gr }}$ Delegato Apostolico.
} 
del Val, le zèle intempestif des ecclésiastiques avait des conséquences vraiment catastrophiques : «... il met en danger imminent la foi de milliers de personnes; il fait hair les sacrements de l'Église, déprécier l'autorité des évêques et du clergé, détruire toute la discipline ecclésiastique; il éloigne encore plus les non-catholiques; il anéantit l'autorité familiale et cause la perte de tellement d'âmes" ${ }^{22}$. Si les choses continuent ainsi, le Canada, au lieu d'être «le rempart et le principal appui de l'Église sur tout le continent nord-américain ... perdra définitivement sa position traditionnelle et ne fera rien à l'avenir» ${ }^{23}$.

Il y avait quand même une lueur d'espoir dans ce tableau assez sombre: c'était l'épiscopat de l'Ontario. Selon le délégué, les archevêques John Walsh de Toronto et James Cleary de Kingston, brillaient par leur doctrine, leur expérience et leur tact. Ils avaient su se gagner l'estime des hommes politiques et des protestants et par conséquent les institutions catholiques étaient florissantes dans cette province. Ces prélats exerçaient une grande influence sur le pays ${ }^{24}$. Comme solution générale aux problèmes religieux du Canada, Merry del Val proposa que les évêques francophones trop souvent portés à se prendre pour l'épiscopat canadien tout entier, n'interviennent dans les délicates questions politico-religieuses, que de concert avec leurs homologues anglophones ${ }^{25}$.

La Congrégation des Affaires ecclésiastiques extraordinaires se réunit quatre fois pour étudier les problèmes soulevés par la mission Merry del Val. Les cardinaux approuvèrent l'analyse et les recommandations du délégué apostolique. Selon eux, la solution définitive de la question scolaire manitobaine se réaliserait, à long terme. Dans l'immédiat, il fallait réclamer, et non refuser, les améliorations à l'accord Laurier-Greenway. Ils rappelèrent aux évêques du Canada les normes du Saint-Siège et celles de leurs propres conciles concernant l'intervention du clergé en politique et blâmèrent à ce chapitre les évêques Michel Labrecque de Chicoutimi et John Cameron d'Antigonish pour leur comportement pendant les élections fédérales de $1896{ }^{26}$. Enfin, après trois requêtes pressantes de Laurier, ils sanctionnèrent en juillet 1898 l'établissement d'une délégation apostolique permanente au Canada.

\footnotetext{
${ }^{22}$ Ibid.

${ }^{23}$ Ibid.

${ }^{24}$ CAEE, Inghilterra, Posizione 161, Merry del Val a Rampolla, 21 maggio 1897.

${ }^{25}$ CAEE, Relazione del Delegato.

${ }^{26}$ CAEE, Ponenza sul Canada, 11 dicembre 1897.
} 
Les cardinaux jugeaient bon cependant d'attendre que les esprits échauffés par la question manitobaine se calment, condition qui assurerait un digne accueil au représentant du Saint-Siège ${ }^{27}$. Le premier ministre canadien redoubla néanmoins ses pressions, ayant aussi recours à ses amis anglais, pour venir à bout de 'ces réticences. C'est ainsi que le Saint-Siège annonça le ler juillet 1899 la nomination de Diodeme Falconio, archevêque de Matera et Acerenza en Italie méridionale, comme premier délégué apostolique permanent au Canada.

\section{$\mathrm{M}^{\mathrm{gr}}$ FALCONIO (1899-1902)}

Falconio dû affronter une situation sensiblement identique à celle de son prédécesseur. D'une part, l'épiscopat francophone se sentait lésé et presque sous tutelle, parce qu'il n'avait pas jugé opportune la création d'un délégation permanente. D'autre part, le gouvernement canadien multipliait ses déclarations de bonne volonté, sans faire beaucoup avancer le dossier des écoles catholiques du Manitoba. L'action du délégué était certes subordonnée à la politique du Saint-Siège, mais il faut aussi prendre en compte qu'il se sentait plus près de Laurier que de Langevin. Il essaya au début de favoriser une entente entre ces deux hommes, en fonction des attentes de Rome, soucieuse de relations harmonieuses entre clergé et autorités civiles. Toutefois, lorsque cette entente échoua, il ne manqua pas d'indiquer ses propres préférences. Ainsi, le délégué commenta favorablement la ré-élection de Laurier en 1900: «... la victoire du parti libéral avec à sa tête un catholique et de la grande majorité des députés catholiques, nous fait espérer de meilleures dispositions à l'égard de notre sainte religion" ${ }^{28}$. D'autre part, il ne voyait en Langevin qu'un être léger, impulsif et, qui plus est partisan: «Je suis attristé de devoir dire que l'animosité de $\mathrm{M}^{\mathrm{gr}}$ Langevin envers ce gouvernement fédéral, auquel il cherche toujours à créer des embarras pour des raisons partisanes, est chose déplorable" ${ }^{29}$. Pendant les trois ans que Falconio se trouva au pays, il ne réussit pas à améliorer le sort des catholiques du Manitoba.

Le délégué eut aussi à examiner des questions plus générales pendant son séjour au Canada dont une avait trait à la discipline ecclésiastique. Falconio ne partageait pas, semble-t-il, les vues pessimistes

\footnotetext{
${ }^{27}$ CAEE, Ponenza sul Canada, luglio 1898.

${ }^{28}$ APFR, NS 195 Rubrica 154, Falconio a Ledochowski, 8 novembre 1900.

${ }^{29}$ CAEE, Inghilterra, Posizione 193 Rubricella 63590, Falconio a Rampolla, 8 maggio 1891.
} 
de Merry del Val. En effet, il caractérisait l'Église canadienne «comme une des parties les plus florissantes du très vaste empire de Jésus-Christ sur terre ${ }^{30}$. Il se félicita aussi du zèle, du dévouement et de la fidélité du clergé, notant également que les ecclésiastiques avaient su respecter les prescriptions du Saint-Siège pendant les élections de $190{ }^{31}$.

Cependant, il y avait, selon lui, un grand besoin d'uniformiser la discipline ecclésiastique. L'Église canadienne devait se doter d'un code qui réglerait les rapports entre les évêques et leurs prêtres et aussi entre le clergé et les fidèles. L'épiscopat s'en remettait trop souvent à son bon vouloir dans la distribution des honneurs et des charges, ce qui engendrait un esprit de complaisance dans le bas clergé. À cet égard, Falconio demanda et reçut, dès les premiers jours de sa délégation, le pouvoir d'entendre les griefs des prêtres contre leurs ordinaires et de les régler soit à l'amiable soit de sa seule autorité ${ }^{32}$. Falconio fit valoir que le Canada n'étant plus un pays de mission, il fallait assurer une place plus stable au curé de paroisse, lui permettant d'accéder aux postes supérieurs «d'après le droit et non d'après la volonté de celui qui gouverne» ${ }^{33}$. Comme remède à ce problème de discipline, le délégué suggéra la tenue de synodes provinciaux réguliers ainsi que la tenue d'un concile national qui favoriserait la bonne entente entre le clergé de diverses nationalités. Soulignons qu'il faisait ici écho à la proposition de Merry del Val qui voulait associer les évêques francophones zélés à leurs collègues ontariens plus modérés.

Falconio décrivit aussi le bas clergé comme autoritaire et porté au luxe. À ce chapitre, il s'étonna que de petites paroisses possédaient des églises qui étaient de "vrais cathédrales» et que les presbytères «ressemblaient à des palais somptueusement meublés». Quoi qu'il en soit, ajouta-t-il, «face aux grandes vertus [du clergé], ces défauts ne sont que peu de chose» ${ }^{34}$.

Il est évident que le Canada à l'époque de Laurier fascina le délégué apostolique. Ce prélat du Vieux Monde fut frappé par l'atmosphère de liberté et de démocratie. À ses yeux, les distinctions sociales n'existaient pas au Canada; ne primait ici que le dollar. Ce trait culturel

${ }^{30}$ APFR, NS 215 Rubrica 154, «La Chiesa Cattolica nel Canada , 3 dicembre 1901.

${ }^{31}$ APFR, NS 215 Rubrica 154, Falconio a Ledochowski, 8 nov. 1900.

${ }^{32}$ APFR, NS 215 Rubrica 154, Ledochowski a Falconio, 22 dicembre 1899.

${ }^{33}$ APFR, "La chiesa cattolica... *

${ }^{34}$ Ibid. 
avait des aspects inquiétants surtout dans le domaine de l'éducation: trop souvent les Canadiens et surtout les Canadiennes poursuivaient des études qui, selon ses vues, ne convenaient pas à leur rang. L'enseignement des collèges classiques au Québec était trop accessible. Les jeunes filles anglophones, pour leur part, se précipitaient vers les académies qui dispensaient des programmes réservés en Italie à la noblesse. Ces études réveillaient chez celles-ci des aspirations sociales qui menaient souvent aux mariages mixtes.

Ce qui impressionna Falconio dans cette époque d'immigration massive, ce fut le mélange d'ethnies au Canada. Suivant la politique établie en 1887 par le Saint-Siège, il se montra sympathique aux aspirations culturelles de ces différents groupes. La nomination de coadjuteurs irlandais dans les deux diocèses du Nouveau-Brunswick avait provoqué l'ire des Acadiens qui réclamaient qu'un des leurs soit élevé à l'épiscopat. Tout en rejetant la création d'un troisième diocèse dans cette petite province, le délégué proposa qu'à l'avenir Rome tienne compte de l'appartenance ethnique des diocésains avant de nommer le titulaire d'un siège vacant.

Les Ukrainiens, pour leur part, posaient un problème très aigu pour Falconio parce qu'ils étaient en proie à une propagnade protestante massive et bien organisée. En effet, les Églises réformées avaient mis à la disposition de ces immigrants toute une gamme de services sociaux qui les aidaient à survivre sur cette terre nouvelle. Le délégué souligna que les Ukrainiens "obstinés comme ils le sont dans leur rite" ${ }^{35}$, "ne sachant ou ne voulant pas s'adapter au rite latin" ${ }^{36}$, avaient besoin de secours spirituels particuliers que les missionnaires en place ne pouvaient leur fournir. De plus, l'Église catholique devait mettre sur pied des comités qui s'occuperaient des besoins matériels des immigrants. Falconio fit remarquer à ce sujet qu'il n'y avait pas d'associations de bienfaisance ou de mutuelles catholiques au Canada.

Quant aux Amérindiens, qui «en général ne sont malheureusement pas fort dans la foi et se laissent facilement séduire» ${ }^{37}$, l'Église devait mettre sur pied une commission qui s'occuperait uniquement de leurs missions.

\footnotetext{
${ }^{35}$ APFR, NS 215 Rubrica 154, Falconio a Rampolla, 20 marzo 1901. «La Propaganda protestante nel Canadan.

${ }^{36}$ APFR, "La chiesa cattolica..."

${ }^{37}$ APFR, "La chiesa cattolica..."
} 
À l'égard des deux principales "nations" canadiennes, le délégué reconnut la supériorité politique et économique des Anglo-protestants. Aux catholiques, qu'ils soient irlandais ou canadiens-français, faisait défaut l'esprit d'initiative et d'entreprise de leurs compatriotes protestants; ils semblaient ressentir encore aujourd'hui le joug des persécutions ancestrales. Les premiers étaient plus solides, plus aguerris aux dangers de la propagande protestante, mieux préparés à réfuter des erreurs doctrinales et plus constants. Quant aux Canadiens français, le prélat répéta tous les lieux communs de Lord Durham: peuple qui auparavant vivait dans l'innocence et la simplicité primitive sous la seule autorité du curé; peuple conservateur qui manquait d'élan et d'initiative et qui était fortement attaché à l'esprit de nationalité. Pour améliorer ce cadre, Falconio misait beaucoup sur l'éducation. Il nota entre autres que la seule université catholique de langue anglaise (sic), l'Université d'Ottawa, n'offrait pas de cours professionnels aux jeunes laïcs. Et pourtant il y avait un grand besoin que ceux-ci puissent étudier dans une institution catholique. Le délégué souligna aussi l'importance d'une presse catholique indépendante pour contrecarrer l'esprit de parti qui régnait au Canada ${ }^{38}$.

\section{$M^{\mathrm{gr}}$ SBARETTI (1902-1910)}

En septembre 1902, le Saint-Siège annonça la promotion de Falconio à la délégation apostolique de Washington et la nomination de $\mathrm{M}^{\mathrm{gr}}$ Donato Sbaretti, ancien titulaire de cette délégation, à Ottawa. Les instructions qu'on lui remit lors de son installation dans la capitale insistaient surtout sur l'importance d'instaurer un climat de confiance et d'intimité entre Rome et les évêques canadiens ${ }^{39}$. Ceux-ci en effet ne devaient voir dans le délégué qu' «un appui à leur mission épiscopale». Sbaretti devait surtout faire un emploi prudent de ses pouvoirs de façon à ne pas porter ombrage aux droits épiscopaux. Dans la question des écoles catholiques au Manitoba, Rome réitérait sa politique de fermeté dans la modération. Le Saint-Siège soulignait la gravité du problème qui pourrait se répercuter dans les autres provinces "où il serait difficile de les (droits catholiques) défendre avec succès, s'ils sont attaqués, après une capitulation trop conciliante au Manitoba" ${ }^{40}$. Rome en outre reconnaissait l'intensité de l'antagonisme qui existait entre anglophones

\footnotetext{
${ }^{38}$ Ibid.

${ }^{39}$ APFR, NS 265 Rubrica 154, Rampolla a Gotti, 18 septembre 1902.

${ }^{40}$ APFR, NS 265 Rubrica 154, Minuto per $M^{\text {gr }}$ Sbaretti.
} 
et francophones au Canada et recommandait à son représentant une attitude de parfaite impartialité. Elle jugeait inopportune la tenue d'un concile national dans le seul but de normaliser les diverses disciplines ecclésiastiques, notant qu'à ce chapitre les anglophones divergeaient trop des francophones. Quant aux querelles entre Canadiens anglais et français à l'égard de l'Université d'Ottawa, le Saint-Siège ne voulait pas donner à cette institution un caractère ethnique. Enfin, Rome répétait ses instructions sur l'ingérence cléricale en politique en vue des élections fédérales ${ }^{41}$.

Les craintes du Saint-Siège quant au sort des écoles catholiques dans les provinces protestantes commencèrent bientôt à se vérifier. Dans la question des écoles du Nord-Ouest, $\mathbf{M}^{\text {gr }}$ Sbaretti dut en effet jouer le même rôle qu'avait joué Merry del Val à peine dix ans auparavant, avec des résultats plus ou moins identiques. Malheureusement, les archives vaticanes sont fermées pour cette période. Nous avons cependant eu accès à des lettres qui révèlent l'état d'âme d'un certain clergé canadienfrançais lors du départ de Sbaretti en 1910. Si l'on s'en tient à ces témoignages, il est clair que l'impartialité parfaite recommandée à Sbaretti par le Saint-Siège s'avéra un idéal bien éloigné de la réalité. Sans ménager ses mots, $\mathbf{M}^{\mathrm{gr}}$ Langevin accusa le délégué de proner un système d'anglicisation à l'intérieur de l'Église canadienne ${ }^{42}$. Il ne tint personnellement responsable de la nomination d'un anglophone, Charles-Hugh Gauthier, au siège d'Ottawa et du francophobe Michael Francis Fallon à London ${ }^{43}$. Louis-Adolphe Pâquet commentat ainsi ces événements: «La race canadienne française aurait-elle démérité de l'Église, et verronsnous une autre race moissonner dans la joie ce que nos missionnaires et nos évêques ont semé dans les larmes»? Le théologien se plaignait en outre que Sbaretti eût choisi un anglophone, A.A. Sinnot, comme secrétaire; "nous en avons souffert", ajoutait-il ${ }^{44}$. $\mathbf{M}^{\text {gr }}$ Sinnot, fit observer un curé québécois, s'était montré trop favorable aux Irlandais ${ }^{45}$.

\footnotetext{
${ }^{41}$ APFR, NS 265 Rubrica 265, Minuto delle Istruzioni per Monsignor Delegato Apostolico nel Canada.

${ }^{42}$ Archives générales de l'ordre des Servites de Marie (AGOSM), A. Langevin et ses suffragants à Pie X, 7 octobre 1910.

${ }^{43}$ AGOSM, Langevin à Alexis Lépicier, 18 octobre 1910.

${ }^{44}$ AGOSM, L. A. Pâquet à Lépicier, 26 novembre 1910.

${ }^{45}$ AGOSM, J. P. Desrosiers à Lépicier, 21 mars 1911.
} 
Il n'est donc pas surprenant que la désignation, en octobre 1910, de $\mathrm{M}^{\mathrm{gr}}$ Pellegrino Stagni, archevêque d'Aquila, près de Rome, ait soulevé tant d'espoir chez ce clergé. Ce n'était pourtant pas ses antécédents qui suscitaient ces émotions. $M^{\text {gr }}$ Stagni avait passé plus de quinze ans à Londres et possédait parfaitement l'anglais. À Rome, il avait été successivement professeur de métaphysique au Collège Urbain, consulteur au Saint-Office et supérieur général de sa communauté, les Servites de Marie ${ }^{46}$. Mais surtout, Stagni était un confrère et intime d'Alexis Lépicier, théologien et maître de plusieurs prêtres canadiens qui avaient étudié à Rome. Lépicier ne cachait pas non plus ses sympathies pour les Canadiens français. Certains ecclésiastiques auraient en fait souhaité sa désignation comme délégué apostolique ${ }^{47}$. Quoi qu'il en soit, un curé montréalais exprima le sentiment de plusieurs lorsqu'il écrivit à Lépicier à propos de Stagni, "amicus amico est amicus" ${ }^{48}$. Un prêtre de Chicoutimi renchérit: "Nous voyons là un indice certain de cette sollicitude toute spéciale de la divine Providence à l'égard des Canadiens français» ${ }^{49}$. Mais il soupçonna aussi une influence plus terre à terre, celle de Lépicier lui-même.

C'est ainsi qu'au début, on ne se gêna pas pour recourir à Lépicier en tant qu'intermédiaire. $\mathbf{M}^{\mathrm{gr}}$ Langevin lui fit part de sa joie face au choix de Stagni, et lui demanda d'intéresser le nouveau délégué apostolique au problème de la nomination de l'évêque de Regina, autre différend qui divisait les clergés canadien-français et irlandais. "Vous pourrez tout expliquer à $\mathrm{Sa}$ Grandeur $\mathbf{M}^{\mathrm{gr}} \mathrm{Stagni}$ et obtenir qu'il nous protège" ${ }^{50}$. L.A. Paquet, agissant à la demande expresse de $\mathbf{M}^{\mathrm{gr}}$ Bégin, pria Lépicier «d'user de votre crédit auprès de $\mathrm{M}^{\mathrm{gr}}$ Stagni en faveur des $\mathrm{Ca}$ nadiens français blessés dans leurs droits et humiliés dans leur fierté nationale" ${ }^{51}$. Un prêtre se fit l'interprète de plusieurs lorsqu'il écrivit à propos de l'action future du prélat: "Il saura déjouer les intrigues qui

\footnotetext{
${ }^{46}$ P.M. Bernardi, Cenno biografico di Monsignor Pellegrino M. Francesco Stagni. (Roma, 1919).

${ }^{47}$ AGOSM, L. N. Bégin à Lépicier, 30 juin 1913; A. Volbars, professeur au Séminaire de St-Sulpice, à Lépicier, 2 mai 1911.

${ }^{48}$ AGOSM, J. C. Geoffrion, vicaire à Hochelaga, à Lépicier, 11 nov. 1910; aussi L. J. T. Lafontaine à Lépicier, 13 déc. 1910.

${ }^{49}$ AGOSM, A. Gaudreault, directeur du petit séminaire de Chicoutimi, 6 nov. 1910.

${ }^{50}$ AGOSM, Langevin à Lépicier, 17 nov. 1910.

${ }^{51}$ AGOSM, Pâquet à Lépicier, 26 nov. 1910.
} 
se trament depuis des années dans le but d'accaparer les sièges épiscopaux - dans le camp irlandais - il percera à jour ces tissus de mensonges que les Irlandais ne cessent de débiter à Rome même" ${ }^{52}$. Un prêtre du collège de Lévis espérait que Stagni couperait court aux intrigues de Laurier qui brandissait de nouveau l'arme de l'ingérence politique contre les enseignants des collèges classiques, partisans de Bourassa ${ }^{53}$.

Pour sa part, le chancelier du diocèse de Sherbrooke voulait que le délégué inculque un esprit plus romain dans le clergé et surtout dans l'épiscopat qui s'obstinait à voir dans tout recours d'un prêtre contre son ordinaire, un crime de lèse-majesté. «Un romain qui viendrait vivre ... dans nos évêchés ... entendrait des conversations, dans les milieux les plus cléricaux, sur l'ingérence indue du Délégué, cet Italien d'Ottawa, dans nos affaires" ${ }^{54}$. Malgré les espoirs qu'on avait mis en lui, Lépicier exerça peu d'influence sur Stagni. Leur correspondance resta assez mince et se limita aux problèmes de leur communauté. De plus, pendant ses six ans à la délégation d'Ottawa, Stagni ne vit que très rarement son confrère qui devint prieur général des Servites.

Stagni arriva à Ottawa le 24 mars 1911. "Il faisait un froid très intense. Nous allâmes à la Délégation en traîneau..." ${ }^{55}$. Son séjour au Canada fut aussi intense que le froid le jour de son arrivée. Il toucha à plusieurs questions brûlantes à une époque où le pays était profondément déchiré et il ne se plaignit jamais d'être oisif. «Depuis Noël (1911) j'ai eu beaucoup à faire: ce sont des questions très graves auxquelles la politique n'est pas étrangère. (Je prépare) à chaque semaine de longs rapports, un, deux ou trois au Secrétariat d'État et à Consistoriale ce qui me fait dépenser à chaque fois trois ou quatre lires à la poste" ${ }^{56}$.

Rappelons quelques-uns des problèmes qu'il eut à affronter. L'antagonisme entre Irlandais et Canadiens français s'intensifia pendant cette période. Le délégué dut s'occuper du Règlement XVII en Ontario et du sort des francophones dans les diocèses irlandais de cette province et d'ailleurs. Cette rivalité se manifesta aussi dans la nomination de nouveaux évêques. D'une part, les Acadiens purent se réjouir de la désignation de $\mathrm{M}^{\mathrm{gr}}$ Le Blanc au diocèse de St-Jean. D'autre part, le démem-

\footnotetext{
${ }^{52}$ AGOSM, Geoffrion à Lépicier, 24 fév. 1911.

${ }^{53}$ AGOSM, Joseph Hallé, Joseph Hallé, professeur au Collège de Lévis, à Lépicier, 11 fév. 1911.

${ }^{54}$ AGOSM, H. A. Simard à Lépicier, 17 déc. 1910.

${ }^{55}$ AGOSM, Stagni à P. M. Bernardi, 5 aprile 1911.

${ }^{56}$ AGOSM, Stagni à Bernardi, 9 febbraio 1912. Les tarifs postaux ont augmenté légèrement depuis ce temps-là!
} 
brement de l'archevêché de Saint-Boniface, le transfert du siège archiépiscopal à Winnipeg et la nomination de $\mathbf{M}^{\mathrm{gr}}$ Sinnot comme premier titulaire furent ressentis comme une gifle par plusieurs Canadiens français. Philippe Perrier écrivit à ce sujet:

La Providence n'a pas l'air de tenir à la survivance de la race française en Amérique. Du moins, elle lui porte de rudes coups. Est-ce pour éprouver sa vitalité? Est-ce pour hâter son trépas. À tout événement Rome a l'air bien décidé de vous étrangler à brève échéance. La division du Diocèse de St-Boniface est une ignominie que le Pape ne connaît pas; mais ses auteurs porteront devant Dieu une lourde responsabilité. ${ }^{57}$

Le prélat fut aussi appelé à s'intéresser aux problèmes de discipline ecclésiastique. C'est lui qui suspendit a divinis les prêtres du Collège de Monnoir dans cette célèbre et douloureuse question. De plus, il eut à trancher dans les différends qui opposaient des évêques et leurs prêtres. Voici par exemple le témoignage d'un abbé de Montréal qui s'était plaint des méthodes autocratiques de $\mathbf{M}^{\mathrm{gr}}$ Paul Bruchési : «Au Canada, un appel au Délégué Apostolique équivaut à une déclaration de guerre contre un évêque et ... en définitive le 'pot de fer' écrase infailliblement 'le pot de terre' ..." ${ }^{58}$.

$\mathrm{M}^{\mathrm{gr}}$ Stagni profita de son poste pour introduire sa communauté au Canada. En effet, il offrit les services des Servites de Marie aux archevêques de Vancouver, d'Ottawa et de Montréal pour la desserte des immigrants italiens de ces villes. Il faut dire que ces prélats n'étaient que trop heureux de se voir offrir une solution durable au problème persistant qu'était celui de trouver des prêtres aptes à desservir les immigrants dans leur propre langue. Stagni, pour sa part, fit pression auprès de sa communauté pour la convaincre d'accepter ces cures. Il vanta les avantages de la législation ecclésiastique du Québec et décrivit en termes louangeurs les conditions matérielles de ces fondations ${ }^{59}$. Ses efforts portèrent fruit.

Cependant, le dévouement, le travail de Stagni, sa science, son affabilité ne suffirent pas dans ce moment de crise. Et les sentiments d'aliénation exprimés par Perrier révélent une déception et une amertume durables face au rôle qu'avait joué jusque-là la Délégation apostolique au Canada.

\footnotetext{
${ }^{57}$ AGOSM, Perrier à Lépicier, 17 nov. 1916; aussi, Edmour Hébert à Lépicier, 25 fév. 1916.

${ }^{58}$ AGOSM, A. Curotte à Lépicier, 5 nov. 1912.

${ }^{59}$ AGOSM, Stagni to Lépicier, 11 September 1911; Stagni à Lépicier, 3 marzo 1912; Stagni à Lépicier, 28 marzo 1912.
} 
Certes, la présence de la Délégation apostolique eut des effets bénéfiques pour l'Église canadienne. Les représentants du Saint-Siège, depuis Conroy jusqu'à Stagni, insistèrent sur l'approfondissement de la culture théologique du clergé. Ils firent pression sur les évêques pour qu'ils mettent fin à l'emploi de jeunes séminaristes dans les collèges classiques. Leurs efforts furent couronnés de succès: on nota à la fin du siècle une nette amélioration dans les études théologiques; et de nombreuses lettres à Lépicier témoignent du fait que, de plus en plus, des prêtres eurent la chance de compléter leurs études à Rome.

On peut dire aussi que les rapports entre le bas clergé et l'épiscopat furent moins laissés à l'arbitraire sous la Délégation apostolique. Il ne faudrait pas sous-estimer l'ampleur de ce problème qui revient trop souvent dans ces quarante ans d'histoire pour être pris à la légère. Remarquons toutefois que $\mathrm{M}^{\mathrm{gr}}$ Conroy y avait été insensible, pis encore, il avait cru que le vrai problème étant le manque d'autorité épiscopale. Sa perception souligne en fait toute la subjectivité de la perception d'un délégué apostolique. Serait-il injuste d'affirmer que le prélat irlandais fut moins sensible à l'autoritarisme des évêques parce que ses victimes étaient les ennemis de $\mathbf{M}^{\mathrm{gr}}$ Taschereau? Et comment se fait-il que Conroy réprouva les rigueurs de $M^{\text {gr }}$ Bourget, alors que Smeulders ne vit que celles de $\mathbf{M}^{\mathrm{gr}}$ Taschereau? $\mathbf{M}^{\mathrm{gr}}$ Merry del Val pour sa part fit l'éloge de $\mathbf{M}^{\mathrm{gr}}$ Walsh pour sa science et ne reconnut pas le même attribut en $\mathbf{M}^{\mathrm{gr}}$ Bégin qui avait son doctorat de Rome et qui possédait sept langues. $\mathbf{M}^{\mathrm{gr}}$ Falconio représenta Laurier comme un excellent catholique, et $\mathrm{M}^{\mathrm{gr}} \mathrm{Lan}-$ gevin, comme un partisan acharné. En fin de compte, ces perceptions sont ce qu'elles sont: on ne voit que ce qu'on veut voir...

Rome et ses représentants avaient une conception nord-américaine du Canada. Ce n'est pas par hasard que des délégués blâmèrent parfois le comportement du clergé québécois en soulignant la mauvaise publicité qui en résulterait aux États-Unis. D'autres comparèrent la préparation apologétique des Irlandais à l'ignorance doctrinale des Canadiens français. Décidément, pour eux, le catholicisme en Amérique passait par la langue anglaise. Ainsi, la capacité de parler anglais était une condition sine qua non de la nomination du délégué à Ottawa ${ }^{60}$. Cette optique

\footnotetext{
${ }^{60} \mathrm{D}$ 'ailleurs Alexis Lépicier nota dans son journal que sa candidature à la Délégation apostolique du Canada fut rejetée parce qu'il était français, AGOSM, Journal Lépicier, 17 novembre 1926. «Ai rencontré Monseigneur Di Maria (délégué apostolique au Canada de 1917 à 1926). La raison (de la décision de la Consistoriale contre la candidature de Lépicier) est que je suis français».
} 
n'était pas non plus en contradiction avec l'ouverture de Rome envers les immigrants. En Amérique, tous avaient leur place, qu'ils fussent Ukrainiens ou Acadiens; mais la langue commune était l'anglais.

Ce point de vue s'opposait à celui du clergé québécois qui en gros possédait, comme l'avait si bien noté $\mathrm{M}^{\mathrm{gr}}$ Merry del Val, une conception nationale de son identité: il était l'Église canadienne. Cette conception était profondément ancrée dans l'histoire. En général, les ecclésiastiques croyaient au caractère particulier de la province de Québec en tant que seul État catholique en Amérique du Nord. Ils furent portés à soupçonner de libéralisme catholique ceux qui refusaient cette vision intégrale de la réalité et voulaient diluer la spécificité catholique du Québec dans le grand ensemble canadien. Mais cette perspective n'était pas introvertie, car elle se doublait d'une vision du Québec (et nous ne parlons pas ici nécessairement de la législature) comme protecteur des droits des minorités catholiques et françaises. Soulignons à cet égard la continuité d'action qui alla de $\mathbf{M}^{\mathrm{gr}}$ Bourget dans les questions de l'enseignement catholique au Canada-Ouest et au Nouveau-Brunswick à $\mathrm{M}^{\mathrm{gr}}$ Bégin dans celles du Manitoba, du Nord-Ouest et de l'Ontario.

Rome rejeta cette façon de voir et interpréta ces initiatives comme de l'ingérence du clergé en politique. En dernier ressort, elle fit plus confiance à Laurier et aux prélats qui avaient une conception «nordaméricaine» de la position de l'Église en tant qu'organisation volontaire ou qui, à la limite, partageaient la vision chauvine des leaders anglophones du Canada anglais. Le lieu d'implantation du siège de la Délégation est fort révélateur à ce sujet. $\mathbf{M}^{\mathrm{gr}}$ Falconio avait à choisir entre Montréal et Ottawa. Cette dernière était certes plus "nord-américaine», mais moins catholique. Ottawa n'en fut pas moins choisie, étant la capitale du pays. Dans tout ceci, le Saint-Siège fit preuve d'un grand réalisme politique et constitutionnel. D'ailleurs lorsqu'on songe au climat de chauvinisme canadien-anglais qui dominait au Canada à l'époque, les options de Rome étaient forcément limitées. Le triomphe de la conception romaine mena inéluctablement à l'aliénation du clergé canadien-français qui se sentit doublement opprimé. Mais la raison du plus fort est toujours la meilleure!

\author{
Roberto PERIN \\ Centre académique canadien \\ en Italie. \\ Rome
}

